\title{
TTR
}

Traduction, terminologie, re?daction

\section{Le vernaculaire noir américain : Ses enjeux pour la traduction envisagés à travers deux oeuvres d'écrivaines noires, Zora Neale Hurston et Alice Walker}

\section{Bernard Vidal}

Volume 7, numéro 2, 2e semestre 1994

Traduire les sociolectes

URI : https://id.erudit.org/iderudit/037185ar

DOI : https://doi.org/10.7202/037185ar

Aller au sommaire du numéro

Éditeur(s)

Association canadienne de traductologie

ISSN

0835-8443 (imprimé)

1708-2188 (numérique)

Découvrir la revue

Citer cet article

Vidal, B. (1994). Le vernaculaire noir américain : Ses enjeux pour la traduction envisagés à travers deux oeuvres d'écrivaines noires, Zora Neale Hurston et Alice Walker. TTR, 7(2), 165-207. https://doi.org/10.7202/037185ar

\section{Résumé de l'article}

Le vernaculaire noir américain : Ses enjeux pour la traduction envisagés à travers deux oeuvres d'écrivaines noires, Zora Neale Hurston et Alice Walker Zora Neale Hurston et Alice Walker font usage dans leurs oeuvres d'une langue autre, d'un sociolecte longtemps dénigré, le vernaculaire noir américain. Cette utilisation va bien au-delà de la simple caractérisation sociale de leurs personnages et constitue un geste contestataire, une revendication et une célébration. Dans ces conditions, la traduction annexionniste qui consisterait à avoir recours à des sociolectes effaçant totalement la négritude et la problématique raciale, tel le langage " paysan ", apparaît comme une véritable mutilation des oeuvres. Il convient donc d'opérer le décentrement du texte-cible en y inscrivant la négritude. Les divers créoles à base française et les variétés du français parlées en Afrique noire peuvent fournir des marqueurs qui, sans relocaliser abusivement le texte-cible, serviront à cette fin.
Tous droits réservés @ C TTR: traduction, terminologie, rédaction — Les auteurs, 1994
Ce document est protégé par la loi sur le droit d'auteur. L'utilisation des services d'Érudit (y compris la reproduction) est assujettie à sa politique d'utilisation que vous pouvez consulter en ligne. 


\section{Le vernaculaire noir américain: Ses enjeux pour la traduction envisagés à travers deux auvres d'écrivaines noires, Zora Neale Hurston et Alice Walker ${ }^{1}$}

\section{Bernard Vidal}

\section{Introduction}

Imaginons, pour le propos de notre article, un jeune traducteur issu de l'Hexagone - donc inévitablement marqué par le classicocentrisme de la culture française -, à qui échoit la tâche de rendre en français le roman de Zora Neale Hurston Their Eyes Were Watching God, dont nous donnons ci-dessous l'incipit:

Ships at a distance have every man's wish on board. For some they come in with the tide. For others they sail forever on the horizon,

1. L'auteur a présenté une première version de cet article (mai 1990) au professeur Chantal Bouchard dans le cadre de ses travaux préparatoires au doctorat. Bernard Vidal est décédé en décembre 1992. Nous avons obtenu l'autorisation de son exécuteur testamentaire pour publier cet article qui a été revisé par Chantal Bouchard. Le roman de Zora Neale Hurston, Their Eyes Were Watching God, a depuis été publié en traduction française sous le titre Une femme noire (Paris, Le Castor Astral, 1993), traduction de Françoise Brodsky. 
never out of sight, never landing until the Watcher turns his eyes away in resignation, his dreams mocked to death by Time. That is the life of men.

Now, women forget all those things they don't want to remember, and remember everything they don't want to forget. The dream is the truth. Then they act and do things accordingly.

So the beginning of this was a woman and she had come back from burying the dead. Not the dead of sick and ailing with friends at the pillow and the feet. She had come back from the sodden and the bloated; the sudden dead, their eyes flung wide open in judgment.

The people all saw her come because it was sundown. The sun was gone, but he had left his footprints in the sky. It was the time for sitting on porches beside the road. It was the time to hear things and talk. These sitters had been tongueless, earless, eyeless conveniences all day long. Mules and other brutes had occupied their skins. But now, the sun and the bossman were gone, so the skins felt powerful and human. They became lords of sounds and lesser things. They passed nations through their mouths. They sat in judgment.

Seeing the woman as she was made them remember the envy they had stored up from other times. So they chewed up the back parts of their minds and swallowed with relish. They made burning statements with questions, and killing tools out of laughs. It was mass cruelty. A mood come alive. Words walking without masters; walking altogether like harmony in a song.

"What she doin' coming back here in dem overhalls? Can't she find no dress to put on? - Where's dat blue satin dress she left here in? - Where all dat money her husband took and died and left her? - What dat ole forty year ole 'oman doin' wid her hair swingin' down her back lak some young gal? - Where she left dat young lad of a boy she went off here wid? - Thought she was going to marry? - Where he left her? What he done wid all her money? - Betcha he off wid some gal so young she ain't even got no hairs - Why she don't stay in her class?"

When she got to where they were she turned her face on the bander log and spoke. They scrambled a noisy "good evenin"" and left their mouths setting open and their ears full of hope. Her speech was pleasant enough, but she kept walking straight on to her gate. The porch couldn't talk for looking. ${ }^{2}$

2. Zora Neale Hurston (1937). Nous nous appuyons sur l'édition publiée par Harper \& Row (1990). 
À la première lecture, notre traducteur ne manquera pas d'être frappé par le dépouillement poétique de la narration, qui, s'il flatte son sens de la pureté, ne lui pose aucun problème auquel sa culture ne l'ait préparé (en dehors des difficultés inhérentes à tout acte de traduction, évidemment). Toutefois, parvenu aux dialogues, il risque d'être un tantinet troublé. Ceux-ci mettent en scène des Noirs américains qui, comme on a pu le constater, parlent une langue fortement idiomatique, dont l'écrivaine a reproduit assez scrupuleusement certains des particularismes phonétiques. Le traducteur, à qui son éthique professionnelle ne permet pas de transiger et conscient de l'enjeu posé, tentera une représentation aussi «fidèle» que possible. Or, quelles solutions sa formation et son passé littéraire lui fourniront-ils? En toute probabilité - et selon notre propre expérience du phénomène -, une série de solutions (géographiques ou sociales) limitées aux seules frontières du pays. Il saura, certes, que son idiome national comprend un certain nombre d'espèces mais les spécifications qu'il aura étudiées (patois, dialectes, parlers, argots) seront irrémédiablement tricolores. Avec pour corollaire que le résultat de son travail pourrait fort bien être la traduction suivante.

- Qu'est-ce qu'elle fiche de retour ici dans cette salopette? Elle a pus de fringues à se mettre sur le dos? - Où est sa robe en satin bleu qu'elle portait quand elle est partie d'ici? - Où est tout le fric que son mari avait gagné, qu'elle a hérité maintenant qu'il a claqué? - Qu'est-ce qu'une nana de quarante berges a besoin de se coiffer comme ça, avec les cheveux qui pendent dans son dos comme une minette? - Où est-ce qu'elle a plaqué le jeune mec avec qui elle s'est tirée? - Cru qu'elle allait se marier? - Où est-ce qu'il l'a larguée? - Qu'est-ce qu'il a fait de tout son pèze? - J'te parie qu'il s'est tiré avec une jeunette qu'a encore même pas de frisettes. - Pourquoi qu'elle reste pas avec ceux de son rang?

Ou celle-ci :

- Qu'est-ce c'est-y qu'elle fait ici dans sa salopette? L'a-t'y pas de quoi se mettre sur le dos? - Où est sa robe en satin bleu qu'elle avait en partant? - Où est le magot que son homme a ramassé et qu'elle a hérité maintenant qu'il est mort? - Pourquoi qu'une femme de quarante ans a besoin de se coiffer comme une jeunesse, avec les cheveux qui pendouillent 
dans le dos? - Qu'est-ce qu'elle a fait de son jeune gars avec qui elle a parti? - Cru qu'elle allait le marier? - Où ce que c'est qu'il l'a laissée? - Et le magot, qu'est qu'il en a fait? - J'croirai bien qu'il a filé avec une jeunette qu'a pas encore de frisettes? - Pourquoi qu'a reste pas avec ceux de son monde?

Le lecteur pourrait penser que nous exagérons. Pourtant, il n'en est rien. Il nous serait aisé de dresser une liste impressionnante d'exemples analogues. Car, face au vernaculaire noir américain ${ }^{3}$ (VNA) la plupart des traducteurs ne voient qu'une seule alternative: l'argot ou le patois. Et le petit scénario que nous venons d'imaginer, que d'aucuns pourraient juger farfelu, n'a en fait rien d'incongru. Il n'est pas même imaginaire. Que l'on remplace le titre Their Eyes Were Watching God par The Color Purple, et nous nous trouvons face à une situation identique, bien réelle, où le traducteur, une traductrice en l'occurrence, forcée à un parti pris a résolument opté pour celui de la neutralisation du dialecte noir en conformant ses ressources aux seules limites de l'Hexagone. Trop à l'écoute de sa culture, elle s'est condamnée à puiser uniquement dans ce fonds les possibilités envisageables, au risque de colorer ses personnages d'une autre teinte; de leur prêter une voix parisienne ou vendéenne chargée d'une autre «nature».

Or, nous soutenons que de tels choix constituent une fausse représentation; qu'ils neutralisent en les déviant les enjeux engagés par les écrivains dans leur langue d'origine; qu'ils entérinent une fois de plus la conception de l'étranger comme un lieu qu'il convient de rapatrier, dans l'acte de traduction, par l'effacement de toute distance culturelle et la promotion de l'identité. Nous postulons en outre qu'en opérant un décentrement - concept que nous empruntons à Meschonnic ${ }^{4}$ - il est possible de parvenir à une

3. Nous empruntons cette expression à William Labov (1976).

4. «Le décentrement est un rapport textuel entre deux textes dans deux langues-cultures jusque dans la structure linguistique de la langue, cette structure linguistique étant valeur dans le système du texte. L'annexion est l'effacement de ce rapport, l'illusion du 
évocation plus proche de l'original, et surtout, que nous pourrons conserver dans leurs propos mêmes la couleur des personnages.

Toutefois, avant de nous engager plus avant dans cette problématique, il serait bon, pensons-nous, de tenter préalablement une évaluation des enjeux socio-idéologiques engagés par les deux écrivaines dans leur représentation du sociolecte ${ }^{5}$ afro-américain.

\section{Les enjeux socio-idéologiques}

Les deux ouvrages que nous avons mentionnés posaient de manière aiguë pour la société réceptrice un même problème: comment

naturel, le comme-si, comme si un texte en langue de départ était écrit en langue d'arrivée, abstraction faite des différences de culture, d'époque, de structure linguistique. Un texte est à distance: on la montre, ou on la cache. Ni importer, ni exporter.» (Meschonnic, 1973, p. 308; italiques de Meschonnic.)

5. Nous tenons tout de suite à nous démarquer du sens que ce terme a pu acquérir en sociocritique, et qui s'apparente plus à nos yeux à la notion de «discours social» (cf. Pierre V. Zima, 1985, pp. 130-150). Notre signification englobe certainement celle qu'en donne Marc Angenot (1979): «l'ensemble des habitudes et des complicités linguistiques d'un petit groupe social: par extension, phraséologie prêtée à un tel groupe dans un récit de fiction: le sociolecte du "petit noyau" des Verdurin, chez Proust) (p. 189); mais, en fait, notre entendement du mot touche moins au contenu qu'à la forme et rejoint la définition de Barthes (1984) dans son article «la Division des langages», à condition toutefois d'éviter le glissement de sens vers "discours social" et "interdiscursivité" que Barthes commet ultérieurement. «Il est temps de donner un nom à ces langages sociaux découpés dans la masse idiomatique, et dont l'étanchéité, pour existentielle que nous l'ayons d'abord ressentie, suit, à travers tous les relais, toutes les nuances et les complications qu'il est licite de concevoir, la division et l'opposition des classes; appelons ces langages de groupe des sociolectes (par opposition évidente à l'idiolecte, ou parler d'un seul individu)» (p. 120). 
traduire le vernaculaire noir américain - à une différence près toutefois, non négligeable pour le traducteur. Le deuxième, qui est aussi le plus récent (1982), est entièrement rédigé dans ce sociolecte qui y occupe de la sorte une place hégémonique. Il s'agit là d'un geste significatif. L'écrivaine Alice Walker s'appuie effrontément sur un langage autre, à forte valeur de «signum ${ }^{6} »$ social, et l'usage qu'elle fait du VNA dépasse la simple caricature habituelle. Il n'est plus question d'objectivation auctorielle à partir d'une position discursive privilégiée, extradialectale; ni de simples insertions de tranches verbales, insérées à coups de guillemets dans un récit autrement très normatif. Le vernaculaire y est constitutif de l'écriture même. Le personnage écrivant s'y investit émotivement, empiriquement, existentiellement: sa destinée est liée à celle de son langage. Il convient d'ajouter à cela que cette altérité langagière qui s'affirme coïncide dans son hors texte avec les différents mouvements politiques d'émancipation des Noirs, des femmes, et qu'elle s'insère dans une littérature qui, sans être totalement nouvelle, n'a acquis droit de cité que depuis peu. Une littérature qui, ainsi que nous le rappelle Sartre (1948) est «lu[e] à la fois par l'opprimé et l'oppresseur, témoignant pour l'opprimé contre l'oppresseur, fournissant à l'oppresseur son image, du dedans et du dehors, prenant avec et pour l'opprimé, conscience de l'oppression, contribuant à former une idéologie constructrice et révolutionnaire.» (p. 289) Dès lors nous comprenons mieux tout le poids du geste d'Alice Walker: peut-on transposer impunément ses Noirs dans l'argot banlieusard parisien ou le patois d'une campagne française?

6. «Lorsque [les] comportements deviennent conscients et voulus, lorsque par eux l'individu affirme, voire affiche et revendique son appartenance à un groupe, ils deviennent ce qu'il est convenu d'appeler [...] un signum, signum de classe, de caste, de corps. Ceci est l'essence de tout argot au sens moderne du mot; dès qu'un groupe vit en société close, dès qu'il prend conscience de sa différence et de sa supériorité, un argot se forme. [...] Plus le sentiment de l'unité et de la cohésion du groupe est fort, plus l'esprit de corps est vif, plus s'affirment les caractères différenciateurs du signum.» (P. Guiraud, 1980, pp. 97-98) 
En imaginant notre scénario précédent, nous nous inspirions effectivement en grande partie de la traduction française de The Color Purple, qui constitue à nos yeux un exemple flagrant de traduction «ethnocentrique». Et, lorsque à son propos nous parlions d'annexion au terroir, nous nous appuyions non seulement sur l'étude du texte d'arrivée (TA), mais aussi, et surtout, sur les propos de la traductrice, Mimi Perrin (1986), qui, aux deuxièmes Assises de la traduction littéraire d'Arles en 1985, justifiait son parti-pris de la façon suivante:

[J'ai dû] me mettre tour à tour dans la peau de Celie l'illettrée, de Sofia la grosse noire qui ne mâche pas ses mots, de Shug la chanteuse de blues au grand cœur qui, elle, a "rroulé sa bosse», mais aussi de l'odieux père, du mari, etc., et transplanter/ transposer en France ces Noirs du sud profond, les rendre crédibles, grâce à des tournures ou des expressions paysannes d'une certaine époque, comme: «Faut que j'la marie», «elle a fauté», «la voilà déjà engrossée», «il l'a mise grosse», «la Celie», (le père», «elle est pas trop fine», «...il me fait d'un ton colère», «une catin et une roulure»... Voilà mes Noirs américains transplantés en plein milieu des Charentes (ou du Poitou, pourquoi pas?)! Il s'agit alors d'improviser comme les jazzmen sur une grille harmonique donnée, de faire «prendre leur chorus» à mes personnages sur la «grille» de base qu'est le texte anglais. (p. 123)

Ces affirmations appellent un certain nombre de remarques. Tout d'abord, que les Noirs sont «transplantés», c'est-à-dire «sortis de la terre pour être replantés ailleurs; transportés d'un pays dans un autre, d'un milieu dans un autre». Le vocable est révélateur. En d'autres termes nous pourrions dire que les Noirs sont tirés d'un milieu socio-économique précis, celui de l'esclavagisme, et de ses dérivés, pour être parachutés dans une campagne où un tel rapport ne peut s'envisager - ce qui ne signifie pas qu'une épreuve de force n'y ait jamais eu lieu: mais en cette société s'affrontaient des Blancs, engagés dans un antagonisme (seigneur-bourgeois-paysan) de nature totalement différente -, de sorte que le déplacement langagier n'a pu manquer d'entraîner corollairement une déterritorialisation de la problématique. D'autre part, en admettant que l'excuse de la transplantation fût acceptable, pourquoi, par 
autophagie, ne pas pousser l'argument jusqu'au bout de sa logique, et transplanter ces Noirs dans un cadre colonial plus proche de celui du TD. Nous pourrions ainsi écrire «Voilà mes Noirs transplantés au milieu de la Côte d'Ivoire, de la Guadeloupe ou de la Louisiane?» Quitte à les transplanter, pourquoi se limiter à l'exiguiité d'un seul territoire? Ce qui nous conduit à notre deuxième remarque.

Nous assistons, une fois de plus, à la manifestation d'un courant de pensée, suffisamment répandu chez certains traducteurs pour en être dérangeant, et qui, par le biais des universaux inhérents à toute peinture sociale, refuse obstinément de voir dans la littérature américaine autre chose qu'une extension de la française. Pour Mimi Perrin le roman se résume ainsi: «Il n'est question ni d'esclavagisme ni de racisme (à un épisode près). C'est une paysannerie, un drame familial, un vrai mélo intimiste» (Perrin, 1986, p. 122). Ces remarques, qui s'insèrent dans une lignée filiatoire qui ne nous est pas inconnue ${ }^{7}$, permettent d'esquiver la problématique réelle

7. Maurice-Edgar Coindreau, le célèbre traducteur de Faulkner écrivait en avril 1960 dans le Princeton Alumni Weekly: «If the country people in Faulkner's work speak a Mississippi dialect, they speak above all as country people do, and nothing else matters. The same reasoning may be applied to Negroes. If Dilsey, the admirable "mammy" of the Compson family in The Sound and the Fury, retains our attention, it is not because of the color of her skin. What makes her a great figure of fiction is the nobility of her character, her qualities of devotion, abnegation, and endurance, all of them qualities which can be rendered in any language without detracting in the least from Dilsey's greatness. All men of my generation have known in the homes of their parents and their grandparents white counterparts of Dilsey. We know how they spoke and this is the thing that concerns us.) (McMillan Reeves, 1971, p. 89). Son disciple, Michel Gresset, renchérit de la façon suivante à propos de Coindreau : " He has always held the view that Dilsey, in The Sound and the Fury, was not so much a Negro woman as the perennial house servant, the kind he had been extremely familiar with since his childhood in Vendée. He, therefore, had no trouble translating her speech, for unlike less tactful translators of Faulkner, he avoided the pidgin 
(raciale) en mettant de l'avant la localisation (la campagne). Tout se réduirait alors à une universalité du rural (issue du présupposé que tous les paysans se ressemblent) grâce à laquelle il ne suffirait plus que d'évoquer le terroir pour circonscrire la difficulté inhérente à la traduction du VNA. Ce qui ne saurait en aucun cas convenir ici. Sartre, à propos d'un autre écrivain américain noir écrivait:

\begin{abstract}
À qui donc Richard Wright s'adresse-t-il? Certainement pas à l'homme universel: il entre dans la notion d'homme universel cette caractéristique essentielle qu'il n'est engagé dans aucune époque particulière et qu'il ne s'émeut ni plus ni moins sur le sort des nègres de Louisiane que sur celui des esclaves romains du temps de Spartacus. L'homme universel ne saurait penser autre chose que les valeurs universelles, il est affirmation pure et abstraite des droits imprescriptibles de l'homme. (Sartre, 1948, p. 100)
\end{abstract}

French of the West Indies for the good old speech of all the Dilseys of the world, whatever their color.) (McMillan Reeves, 1971, Introduction). La filiation est on ne peut plus nette.

De M.-E. Coindreau à Mimi Perrin en passant par M. Gresset, nous pouvons dresser un remarquable inventaire d'affirmations analogues. Le travail de madame Perrin s'insère diligemment dans cette école qui, évidemment, s'inscrit en faux contre les théories de Meschonnic et de Berman, dont nous nous réclamons. La lecture de la Couleur pourpre en français tend à entériner cette peur du métissage que dénonce Berman, précisément. «Toute culture résiste à la traduction, même si elle a besoin essentiellement de celle-ci. La visée même de la traduction ouvrir au niveau de l'écrit un certain rapport à l'Autre, féconder le Propre par la médiation de l'Étranger - heurte de front la structure ethnocentrique de toute culture, ou cette espèce de narcissisme qui fait que toute société voudrait être un Tout pur et non mélangé.» (Berman, 1984, p. 16) Car c'est bien de cela qu'il s'agit. La traduction, dans la saisie des langages sociaux, ne se résout pas à admettre une coloration graphique autre, héréditairement confinée à une couleur locale quasi obsessionnelle: la Vendée, les Charentes, le Poitou. 
En somme, la négritude, le geste contestataire de l'écrivaine noire ont été estompés, et réintégrent sagement un monde paysan «où la Celie» peut vivre en paix, exilée, loin de son «sol-de-langue» (Berman, 1985, pp. 67-81), à l'abri de toute «économie de l'esclavagisme». D'ailleurs les propos de Mimi Perrin montrent bien que cette problématique n'est pas suffisamment perçue en français puisqu'elle n'y signale jamais les enjeux existentiels ou sociopolitiques appelés par le choix stylistique de l'auteure ${ }^{8}$,

8. L'article entier de madame Perrin, intitulé «Improviser comme les jazzmen», constitue en fait une remarquable illustration des théories annexionnistes. À aucun moment il n'y est fait allusion au devenir historique de la communauté noire américaine, ni au fait que le français a lui aussi constitué une langue esclavagiste. La fin de son article sombre dans l'extravagance narcissique : «Je voudrais conclure sur l'importance capitale qu'il faut accorder à la musique dans le dialogue, précisément parce que celui-ci est véhiculé par la voix, premier et unique instrument naturel. [...] Le groupe vocal les Double Six que je dirigeais dans les années soixante reproduisait un grand orchestre de jazz, chaque chanteur s'identifiant à un instrument. Pour faciliter cette symbiose, j'imaginais des paroles qui «traduisaient» le plus fidèlement possible la sonorité, le phrasé, les attaques, l'articulation des différents instruments, tout en racontant une histoire.» (Perrin, 1986 , p. 123). Nous sommes loin de la traduction. Certes, nous ne nierons pas que l'intérêt d'un dialogue repose pour une grande part dans son rythme et sa cadence; que plus il est «enlevé», plus il sonne «vrai»; que "l'on juge un traducteur à ses dialogues», selon la formule de M.-E. Coindreau. Toutefois, les propos de Mimi Perrin ainsi que ceux de plusieurs autres participants aux Deuxièmes Assises accordent, nous semble-t-il, une trop grande part à la sonorité, à la musicalité au détriment de la matière langagière dans ce qu'elle a d'essentiel. Leur souci du rythme les entraîne sur une voix connexe, concomitante à notre problématique certes, mais secondaire à celle-ci. On ne se soucie plus de la négritude ou, si négritude il y a, elle serait suffisamment représentée dans le rythme. Mais suffit-il de susciter une musicalité, un «jazzé» verbal pour rendre en traduction les Noirs américains? 
n'hésitant pas à affirmer qu'il n'y est pas question d'esclavagisme. Si elle a partiellement raison pour ce qui est du récit pris au niveau dénotatif - comme une succession d'événements et de leurs diverses relations d'enchaînement, d'opposition et de répétitions -, c'est toutefois refuser la position du récit considéré comme l'acte de narrer pris en lui-même. L'économie de l'esclavagisme, refusée par la traductrice, n'a pas lieu de s'inscrire fonctionnellement dans le texte puisqu'elle y figurait antérieurement, et de façon urgente, dans l'acte préalable de l'écriture. De sorte que la narration en effectuant ce déplacement du personnage au narrateur, en octroyant à la Forme la valeur consciente d'un signe, place par contrecoup le lecteur devant une alternative immédiate: celle d'accepter ou de refuser l'écriture. S'il la refuse, c'est toute la spécificité du groupe représenté qui se trouve rejetée. S'il l'accepte, il endosse toutes les prémisses du choix puisque cette Forme, devenue signe social, acquiert une «valeur d'épargne». Elle fonctionne «comme un signal économique grâce auquel le scripteur impose sans cesse sa conversion sans en retracer jamais l'histoire ${ }^{2}$ ». Le corollaire étant que c'est dans cet instant névralgique que le récepteur, noir ou blanc, renoue avec la problématique de l'esclavagisme. Mais, si le lecteur potentiel peut rejeter les prémisses et refuser la lecture, le traducteur, lui, qui a acquiescé à la tâche, endosse inévitablement toutes les prémisses de l'écrivaine.

Et dans le cas qui nous intéresse celles-ci sont de taille.

Tout d'abord, de l'aveu même de Zora Neale Hurston, une des motivations de l'utilisation du VNA (résolument entérinée par Alice Walker) visait à prouver que celui-ci, loin d'être une simple négligence langagière due à une quelconque déficience d'apprentissage, représente bel et bien dans sa forme stylistique l'âme du groupe dont il est le moyen d'expression; que ses écarts de

9. Roland Barthes (1953, p. 23) parle des écritures intellectuelle, marxiste, féministe, léniniste, etc. Nous appliquons le même raisonnement pour le choix d'Alice Walker (et de Zora Neale Hurston), que nous percevons comme une écriture politique. 
conjugaison ne relèvent pas que de l'aberration grammaticale mais répondent à une nécessité expressive intrinsèque à ses locuteurs ${ }^{10}$; que son emploi est rendu inévitable par les lacunes du bon usage qui ne permet pas l'expression des mêmes nuances temporelles; bref, que le VNA constitue au même titre que l'anglais standard une forme d'expression à part entière, aussi riche et variée, forme à laquelle il serait temps d'allouer ses lettres de noblesse en la constituant en littérature.

In her indication that black speech was not a separate "language," but a dialect that drew from its linguistic pasts the ideas and structures of the languages that contributed to its viability, Hurston contradicted the conceptualization of black language as an unsuccessful (due to the speaker's inability and the language capacity's smallness) attempt at mimicry of standard language patterns. In addition, the rationale for cultural deficiency, a postscript to this notion of mimicry and truncation, falls disabled as she uses dialect for the vehicle to express the complex consciousnesses of her people. Her note in Negro that the dialect appears and sustains itself because the language of the West has such sterility that it cannot contain the adorned consciousnesses of

10. Karla Holloway à propos de la forme verbale «done» analyse son emploi à la fois dans le VNA et chez Hurston. «Its use suggests an intensity not possible with the simple past and not equivalent to the standard English perfect tense. This is illustrated in structures that permit the standard English had in the perfect aspect of "dat mosquito had done cleaned up ten acres" and also done in the perfect aspect of "Ah done dropped my hammer". $\mathrm{Had}$ and done cannot both be perfect. The dialect indicates, again, that the form carries an additional lexical marker. Done acts as an intensifier when it appears in addition to the perfective or progressive features. It is as if the speaker must indicate that no matter what accoutrements of the standard he uses, his own dialect's cultural markers must be included for the semantic sense he wants to convey. His reality governs the linguistic community in his words. He communicates more than grammatical structure - he communicates cultural sense.) (Holloway, 1987, p. 96) 
black folk is an affirmation of the linguistic power that she understands exists within this dialect. It is, as well, an affirmation of the power she saw in herself, a speaker of the dialect. (Holloway, 1987, p. 115)

Zora Neale Hurston reviendra plusieurs fois sur cette différence fondamentale qu' elle perçoit entre l'anglais standard et le parler des Noirs qui, à ses yeux, est plus imagé, plus exubérant, et d'une remarquable énergie métaphorique ${ }^{11}$. Loin d'elle, donc, l'idée du vernaculaire comme un simple code exotique par lequel on rehausserait la couleur locale de l'œuvre. L'utilisation qu'elle en fait ne se limite pas à une unique visée intratextuelle, elle déborde considérablement le champ de la fiction et de la création

11. Zora Neale Hurston a en fait pris une position polémique opposée à celle qui a longtemps prévalu. Ainsi de ces remarques extraites de The Sanctified Church: «The stark, trimmed phrases of the Occident seem too bare for the voluptuous child of the sun") (1981, p. 53); «In the mouth of the Negro the English language loses its stiffness, yet conveys its meaning accurately. "The booming bounderies of this whirling world" conveys just as accurate a picture as mere "bounderies", and a little music is gained besides.) (p. 81)

Cette énergie n'était pas non plus inconnue des Blancs. Dejà, à propos du dialecte utilisé par Joel Chandler Harris dans Uncle Remus, il était possible de lire le commentaire qui suit: «If [...] the language in which the legends have been framed in the Constitution has given vivid hints of the really poetic imagination of the Negro; if it has embodied the quaint and rugged humor which was the most characteristic; if it has made clear a certain picturesque sensitiveness - a curious exaltation of mind and temperament - if this has been done, then the attempt to reproduce plantation dialect has been measurably successful.» (Cousins, 1968, p. 111) 
romanesque, pour revêtir une valeur sociale à part entière, non dénuée d'intentions contestataires ${ }^{12}$.

En effet, ultimement, les écrivaines (Hurston d'abord, puis Walker ensuite) visent à l'instauration d'un sédiment culturel original, d'un réseau qui, par ses occurrences, se constituerait en héritage tangible. Les écarts qui lui sont inhérents favoriseraient en même temps un ébranlement de l'hégémonie normative, ce qui ne serait pas pour déplaire aux intéressées ${ }^{13}$. Il n'y a pas à remonter très loin dans le temps: reportons-nous aux années 60 par

12. C'est dans cette intention contestataire que Hurston se distingue, comme nous le verrons un peu plus loin. Car la représentation du VNA n'avait rien de nouveau en elle-même dans les années 30 . Pensons à Uncle Tom's Cabin, la série des Uncle Remus, ou même certains textes de Faulkner, Dos Passos, etc. Toutefois, il s'agissait dans ces cas-là de Blancs mettant en scène des Noirs, selon une perception, une problématique et une poétique différentes. Hurston tient à se démarquer autant par ses thèmes que par son style du «plantation dialect» qui, effectivement se limitait trop à la plantation, ou à la cuisine des Blancs.

13. Ces intentions rejoignent en fait celles qui ont été énoncées par $\mathrm{G}$. Lane-Mercier (1990) à propos de l'insertion des sociolectes : «Historiquement marquées vis-à-vis d'une forme perçue comme normative, ces inscriptions se posent en tant qu'infractions et, comme telles, portent atteinte tantôt à l'institution littéraire (qui a toujours prôné la standardisation de l'orthographe ainsi que tout un code de «bienséances verbales» - en témoigne, jusqu'au mouvement naturaliste, l'exclusion des parlers populaires et argotiques du domaine littéraire), tantôt au scriptural, senti comme supérieur au code oral parce que plus homogène (voir le refus d'enregistrer les «ratés» de la communication : bafouillage, hésitations, «bruits» non linguistiques), tantôt encore à un discours dominant précis.» (p. 45) 
exemple $^{14}$, aux débats suscités par le VNA - son statut dans la linguistique, sa place dans les lettres et dans l'enseignement, son impact sur la culture américaine - pour mieux saisir toute l'importance de cet héritage qui, bien qu'il soit nié par la majorité, réussira néanmoins s'il est fondé en culture, à devenir pour la communauté qu'il représente une des preuves de son altérité. Comme le remarque G. Lane-Mercier, «l'inscription romanesque d'éléments dialogaux ouvertement "autres" soulève invariablement la dialectique de la "distinction linguistique" et de la "forme légitime"» (1990, p. 45) et, pourrions-nous ajouter, celle de la dialectique entre le «moi» et «l'autre» puisque, le «moi» étant constitué de part en part du langage, c'est tout le sujet humain qui s'engage dans la parole et se constitue à travers elle (Barthes, 1984, p. 126).

Aussi verrons-nous les écrivains et les critiques afroaméricains partager un même souci: celui d'appréhender et de fixer sur le papier, avant qu'il ne soit trop tard, ce parler autre, cette histoire verbale en suspension, seule possibilité de retrouver un moi fragmenté, incertain, au sein d'une société dont le dessein a longtemps été de le nier. Zora Neale Hurston (1935) pour sa part a directement contribué à la préservation de ce passé par son travail d'ethnologue, et le résultat de ses recherches Mules and Men, qui vient d'être republié, atteste cet effort. Effort qui ne fut ni superflu ni vain si l'on considère, à l'inverse, ce qu'il est advenu du créole français de Louisiane, le gombo, que l'absence d'une préoccupation analogue aussi énergique et concertée a mené à une quasi extinction, fort regrettable. Les vestiges que nous en ont laissés quelques rares auteurs sont pour le moins parcimonieux et ne permettent pas, hélas, d'établir en français un système/patrimoine correspondant, aussi riche et varié. Mais nous aurons l'occasion de revenir sur ce point.

14. Voir à ce propos McCrum, Cran, and MacNeil (1986, pp. 195233); J.L. Dillard (1973), le premier chapitre en particulier : «Black English and the Academic Establishment»; et Cheryl A. Wall, dir. (1989), dont nous nous inspirons largement pour les remarques qui suivent. 
Retenons pour l'instant l'intention essentielle, et avouée, de faire du VNA le témoin d'une histoire.

Toutefois, pour une écrivaine noire, l'inscription du vernaculaire peut se doubler d'une intention autre qui, pour être plus subtile n'en est pas moins tout aussi polémique. En effet, nous savons que 1) aux États-Unis la linguistique a longtemps refusé de prendre en compte le substrat africain du VNA; 2) la littérature américaine n'a pas immédiatement accepté dans son giron la production des auteurs noirs; 3) que même après que ces derniers ont eu acquis droit de cité aux côtés de Faulkner, Fitzgerald, Hemingway, Melville et les autres, ce cercle - à majorité masculine - n'a pas nécessairement fait preuve de la même réceptivité pour ses équivalents féminins; 4) enfin, que le monde universitaire dans ses programmes et les médias dans leurs critiques ont longtemps boudé les œuvres de ces mêmes écrivaines noires ${ }^{15}$. De sorte qu'une auteure telle que Zora Neale Hurston a dû affronter un quadruple handicap sur le chemin de la reconnaissance: économique d'abord, sexuel et spatial ensuite (en disputant à ses homologues masculins un espace médiatique qu'ils étaient d'autant moins disposés à partager avec elle qu'ils avaient eu plus de mal à l'occuper), puis racial.

Or, cette éviction systématique, autant du monde artistique blanc que plus tard du cénacle des écrivains afro-américains a, d'une part, instauré chez ces écrivaines une conscience aiguë de leur différence, mais d'autre part, et paradoxalement, abouti à un renversement imprévisible de la problématique, les intéressées ayant

15. «At that time [1977], most feminist journals were practically all white publications; their content dealt almost exclusively with white women as if they were the only women in the United States. The extent to which the mid-twentieth-century women's movement was becoming like its nineteenth-century predecessor, infected by racism seemed all too clear, and the split between a black and a white women's movement that occurred in the nineteenth century seemed to be repeating itself.» (Christian, 1989, p. 62). 
choisi de miser sur cette différence plutôt que de tenter de l'effacer. De sorte que si elles ont commencé au nom de la justice par en faire leur cheval de bataille, depuis, elles s'en réclament ouvertement, refusant de rejoindre les rangs des féministes blanches autant que ceux des écrivains noirs; le corollaire en est que l'exclusion tant dénoncée et condamnée, de forcée et inévitable qu'elle était à l'origine, est maintenant revendiquée comme une extériorité privilégiée offrant une perspective à la fois intérieure et extérieure, jouant conjointement sur la normalité et la marginalité. C'est à la lisière de cette frontière que les écrivaines - et les critiques espèrent trouver leur spécificité, leur émancipation, en insistant sur le caractère particulier de leur écriture ${ }^{16}$.

16. «As Bakhtin [...] suggests, fusion with the (dominant) Other can only duplicate the tragedy or misfortune of the Other's dilemma. On the other hand, as Gadamer makes clear, "there is a kind of experience of the "Thou" that seeks to discover things that are typical in the behaviour of [the other] and is able to make predictions concerning another person on the basis of [a commonality] of experience." To maintain this insider/outsider position, or perhaps what Myra Jehlen calls the "extra-terrestial fulcrum" that Archimedes never acquired, is to see the other, but also to see what the other cannot see, and to use this insight to enrich both our own and the other's understanding.

As gendered and racial subjects, black women speak/write in multiple voices - not all simultaneoulsy or with equal weight, but with various and changing degrees of intensity, privileging one parole and then another. One discovers in these writers a kind of internal dialogue reflecting an intrasubjective engagement with the intersubjective aspects of self, a dialectic neither repressing difference nor, for that matter, privileging identity, but rather expressing engagement with the social aspects of self ("the other[s] in ourselves"). It is this subjective plurality (rather than the notion of the cohesive or fractured subject) that, finally, allows the black woman to become an expressive site for a dialectics/dialogics of identity and difference.» (Henderson, 1989, p. 36) 
Yet the objective of these writers is not, as some critics suggest, to move from margin to center, but to remain on the border of discourse, speaking from the vantage point of the insider/outsider. (Henderson, 1989, p. 36)

Or, Zora Neale Hurston, anticipant ces positions contemporaines, avait déjà opté pour cette extranéité limitrophe, tant dans sa vie que dans son œuvre. Cherchant sa voix plutôt que sa voie, elle avait trouvé dans le VNA le moyen d'inscrire son originalité, d'affirmer son indépendance, de «talk back», existentiellement d'abord face au monde littéraire noir de l'entredeux-guerres, symboliquement ensuite dans la vie de ses héroünes la réappropriation d'une identité exigeant celle de la parole ${ }^{17}$. Ainsi, admise dans les cercles intellectuels de la Renaissance de Harlem, Hurston n'a jamais totalement adhéré à leur credo qu'elle percevait comme une forme de misérabilisme larmoyant - à la colère évidemment des tenants du sacerdoce, Richard Wright en tête. Ceux-ci ne lui pardonneront pas en retour ses choix qu'ils jugeront trop individualistes, trop folkloristes, insuffisamment politisés, et corroborant l'image traditionnelle du Noir telle qu'elle s'était matérialisée par l'intermédiaire de l'école dite de la "couleur locale» dans la littérature américaine des cinquante années antérieures. Eux qui cherchaient par tous les moyens à échapper aux stéréotypes où les avait confinés la société des Blancs et à stigmatiser les préjugés,

17. Ce sera même un des thèmes centraux de toute l'œuvre de Hurston (et plus tard de Walker) que ce moment clé où la femme noire jusqu'alors opprimée, violentée, reprend son destin en mains et acquiert le droit de parler après une confrontation orale violente avec le mari, principal tenant de l'autorité. Il ne sera peut-être pas indifférent à cet instant-là de constater la langue dans laquelle cette confrontation s'effectue, tant de façon symbolique au niveau intratextuel, qu'au niveau extratextuel, existentiellement. Car un parallèle certain existe entre une Celia ou une Janie, deux héroïnes qui parviennent à maturité le jour où elles se saisissent du mot, et (répondent», brisant le silence opprimant où les réduisait leur condition d'épouse, et l'écrivaine qui décide de ne plus se conformer et brise à l'unisson le carcan de la norme, se saisissant du VNA et l'inscrivant de manière hégémonique dans son œuvre. 
admettaient mal une écriture inverse ni que cette écrivaine, évoluant à la périphérie, fût fière de sa négritude et plus préoccupée de jouir de sa culture, de son texte pourrions-nous dire, de savourer la richesse de l'imaginaire noir, que de dénoncer le racisme; qu'il était plus important à ses yeux de démontrer la valeur d'un bien que l'on possède que le vice de celui des autres; qu'elle participait à la même entreprise qu'eux, mais à sa manière, vantant plutôt que dénonçant, préférant le Sud profond au Nord urbain alors en vogue, cédant la parole à une paysannerie haute en couleurs, dont la joviale volubilité et le dialecte imagé n'avaient jusqu' alors jamais été représentés avec un tel plaisir; qu'elle voulait être une artiste qui, pour la première fois, créerait un monde qui ne fût pas la banale copie de celui des Blancs $^{18}$.

Nous saisissons mieux maintenant pourquoi Zora Neale Hurston, par son octroi conscient de la parole aux Noirs du Sud, par son usage savoureux du VNA, par le parcours de ses héroïnes, est considérée par les écrivaines afro-américaines des années 80 comme leur précurseur (surtout si l'on dresse un parallèle avec l'attitude des mêmes détracteurs de Hurston envers le VNA et les femmes); pourquoi le VNA, qui se situe à la confluence de tous ces courants tant individuels que collectifs, n'est pas, et en fait n'a jamais été, qu'une banale forme créolisante, mais qu'il constitue le passé, la ressource, la matrice de l'esprit d'un peuple, et qu'à ce titre, il ne saurait se satisfaire d'une traduction qui le blanchit et l'édulcore.

18. Ainsi à propos de la réception des textes de Hurston par les Noirs urbanisés du Nord, Alice Walker précise : «No matter how they read the stories Zora had collected, no matter how much distance they tried to maintain between themselves, as new sophisticates, and the lives their parents and grandparents lived, no matter how they tried to remain cool toward all Zora revealed, in the end they could not hold back the smiles, the laughter, the joy over who she was showing them to be: descendants of an inventive, joyous, courageous, and outrageous people; loving drama, appreciating wit, and, most of all, relishing the pleasure of each other's loquacious and bodacious company.» (Walker, 1984, p. 85) 
Finally, we learn how dialect can accomplish for language what poetry accomplishes for prose. In the same manner that a line of poetry loses force, beauty and its concise construction and imagery when translated into prose, dialect loses those qualities when translated into the standard. Dialect and poetry both intensify. Their adornment and special structures are their speakers'conscious manipulations of language to render their experiences as they have felt them. It is a mimetic act - an effort to re-create, through the word, the experiences of a culture. An informed and critical reading audience must be aware of the effort as well as willing to give close enough scrutiny to the text itself to interpret the message of an author such as Hurston, who has specialized her language through the structures, the sounds and the sense of her dialect. (Holloway, 1987, p. 97)

\section{Les enjeux pragmatiques}

Le tour d'horizon des pages précédentes - aussi sommaire soit-il a démontré la nécessité où se trouvait la traduction de prendre conscience, en français, de l'altérité langagière des Noirs, et donc de parvenir à un moyen d'évoquer leur individualité - autrement que par le stéréotype.

Ce besoin maintenant établi, revenons à nos textes de départ et considérons l'aspect proprement pragmatique de la problématique $^{19}$. En plus du court segment en VNA qui a servi d'amorce à ce travail et que nous nommerons $\mathrm{H}-1$, nous aimerions introduire deux autres extraits, successivement $\mathrm{H}-2$ et $\mathrm{W}-1$. Ainsi, au chapitre II de Their Eyes (p. 8), dans une longue diatribe dont nous donnons ci-dessous le premier paragraphe, Janie raconte son enfance à son amie.

19. Nous travaillerons essentiellement pour des raisons qui deviendront vite évidentes sur Their Eyes Were Watching God de Zora Neale Hurston, ainsi que sur The Color Purple d'Alice Walker. Toutefois, nous n'hésiterons pas à nous livrer à de brèves incursions dans d'autres textes pour les besoins de notre argument. 


\section{H-2}

where to start at.

"Ah know exactly what Ah got to tell yuh, but it's hard to know

"Ah ain't never seen mah papa. And Ah didn't know 'im if Ah did. Mah mama neither. She was gone from round dere long before Ah wuz big enough tuh know. Mah grandma raised me. Mah grandma and de white folks she worked wid. She had a house out in de back-yard and dat's where Ah wuz born. They was quality white folks up dere in West Florida. Named Washburn. She had four gran'chillun on de place and all of us played together and dat's how come Ah never called mah Grandma nothin' but Nanny, 'cause dat's what everybody on de place called her. Nanny used to ketch us in out devilment and lick every youngun on de place and Mis' Washburn did de same. Ah reckon dey never hit us ah lick amiss 'cause dem three boys and us two girls wus pretty aggravatin', Ah speck."

De la même façon, dans la deuxième lettre de The Color Purple (p. 3), Celie raconte la mort de sa mère.

\section{W-1}

Dear God,

My mama dead. She die screaming and cussing. She scream at me. She cuss at me. I'm big. I can't move fast enough. By time I git back from the well, the water be warm. By time I git the tray ready the food be cold. By time I git all the children ready for school it be dinner time. He don't say nothing. He set there by the bed holding her hand an cryin, talking bout don't leave me, don't go.

She ast me bout the first one Whose it is? I say God's. I don't know no other man or what else to say. When I start to hurt and then my stomach start moving and then that little baby come out my pussy chewing on it fist you could have knock me over with a feather.

Don't nobody come see us.

Ces deux extraits, choisis arbitrairement, n'ont comme seule correspondance que de se trouver en deuxième place (deuxième chapitre, deuxième lettre), d'être rédigés en vernaculaire, et de représenter l'un et l'autre une forme de confidence, verbale dans le premier cas, écrite dans le second. Distinction qui n'invalide en rien leur voisinage car, même s'il relève de l'épistolaire, le second extrait marque indubitablement sa dette au verbal, par son style et son rythme. 
Après leur lecture, trois remarques s'imposent: tout d'abord, qu'il est possible, pour quiconque possède une connaissance courante de l'anglais véhiculaire, de comprendre sans trop de difficultés les propos des deux jeunes femmes, cela en dépit de leurs particularismes propres ${ }^{20}$. Ensuite, que le choix des marqueurs linguistiques ne fait intervenir que les niveaux phonétique et syntaxique, excluant le sémantique. Enfin, que, bien que provenant du même groupe social et du même lieu, le Sud rural, leurs propos affichent néanmoins des divergences sensibles dans le choix des marqueurs mobilisés. À cela nous pouvons proposer deux explications: d'une part les variations régionales, toujours possibles, ne serait-ce que dans la transformation du «I» en «Ah»; mais surtout, et c'est là à nos yeux le point essentiel, la concrétisation du phénomène de «clôture», inévitable lors de l'inscription romanesque du verbal, la parole fictive demeurant assujettie à sa littérarité, de sorte que:

tout dialogue romanesque ne contient qu'un nombre plus ou moins limité de particularités pragmatico-linguistiques caractéristiques $d u$ parler réel représenté, et ce en raison d'un tri tout à fait conscient effectué par l'auteur parmi ces dernières. Soulignons que ce tri peut opérer à de multiples niveaux et, dans tous les cas, donne lieu à des réaménagements divers qui «réduisent» le taux de réalisme des passages dialogués. Il en résulte ce que nous appelons un effet de clôture, dans la mesure où les réseaux dialogaux intratextuels se trouvent réduits, incomplets, appauvris relativement au modèle réel senti comme nettement plus riche et «ouvert». De plus, les traits pragmatico-linguistiques effectivement retenus par l'auteur ont tendance à se systématiserau sein du texte et, ce faisant, à engendrer des réseaux de redondances. (LaneMercier, 1990, p. 61; nos italiques.)

20. En fait la transcription de leurs propos nous semble plus facile que chez bien d'autres auteurs, blancs en l'occurrence, tels que Mark Twain, Harriet Beecher Stowe, Dos Passos, Faulkner, et certainement Joel Chandler Harris. 
Cet arbitraire dans le nombre et le choix des indices sociolectaux souligne bien à nos yeux l'aspect factice de la mimésis verbale qui, si elle tend vers une évocation aussi plausible que possible du réel, n'en est jamais pour autant sa représentation parfaite ni absolue. N'oublions pas non plus que les sociolectes vivants ne sont, de par leur nature, que des formes souvent transitoires, fluctuantes, rarement figées ou lexicalisées, et que leur transcription romanesque varie forcément selon l'oreille de l'écrivain. De là l'éventail relativement vaste de divergences (de clôtures?) entre les textes d'auteurs variés: un bref coup d'œil aux dialogues en VNA dans les auvres de Richard Wright, James Baldwin, Chester Himes, Faulkner, Dos Passos, Mark Twain, etc., prouvera aisément la variété du mimétisme linguistique.

Pourtant, en dépit de cette potentialité de dispersion, le VNA littérarisé n'en conserve pas moins toujours son accessibilité, même dans les cas limite comme avec Joel Chandler Harris ou Faulkner. Ce dernier semble parfois pousser aussi loin qu'il le peut la liberté graphique, ainsi qu'en atteste le fragment ci-dessous:

"All right," Dilsey said, "All right, here I is. I'll fill hit soon ez I git some hot water." [...] "Put hit down dar en g'awn back to bed." "You put hit down and g'awn back to bed " [...] "I'll have de fire gwine in a minute, en de water hot in two mo." [...] "I'll fix hit in a minute," she said. "Luster overslep dis mawnin, up half de night at "dat show. I gwine build de fire myself. Go now, so you wont wake de others twell I ready." [...]

Twusn't none of Jason money he went on," Dilsey said. "Dat's one thing sho." $[\ldots]$

"Dont you dare come in dis do widout a armful of wood," she said.

"Here I done had to tote yo wood en build yo fire bofe. Didn't I tole you not to leave dis place last night befo dat woodbox wus full to de top?" (Faulkner, 1954, pp. 333-335)

La représentation faulknérienne du verbe de Dilsey, la servante noire de The Sound and The Fury, reçoit dans le chapitre IV, le dernier, une charge sociolectale très prononcée. Ses paroles («Here I is», «fill hit soon ez I git», «Twusn't none of..», «Here I 
done had to tote yo wood en build yo fire bofe.»), adoptent un modèle régulier d'altérations, et dans la dernière phrase en particulier, les marqueurs syntaxiques et phonétiques touchent pratiquement un mot sur deux (Here I [omission] done [omission] to [tote] [yo] wood [en] build [yo] fire [bofe]). Cette alternance sur les structures de surface, procédé extrême dont la lecture exige un effort de décodage, nous mène, pensons-nous, au seuil au-delà duquel les propos de Dilsey deviendraient illisibles. De sorte qu'en dépit d'une apparence de transgression, en dépit d'une déviance poussée à sa limite, il est important de constater que le texte a cependant conservé son accessibilité - qu'il est resté lisible dans la mesure où, conscient que «la parole fictive n'oublie jamais sa littérarité», il a su se restreindre et préserver sa lisibilité ${ }^{21}$. Lisibilité maintenue aussi par un vocabulaire se réduisant à des termes usuels, familiers qu'aucun argot ni jargon ne viennent perturber. Tous les extraits cités sont à l'unisson, et tout à fait représentatifs de la tendance du VNA à se distinguer avant tout, dans la fiction autant que dans la

21. On se reportera pour ce qui est des problèmes de la lisibilité à l'étude déjà citée de G. Lane-Mercier, dont nous nous inspirons fortement pour notre cheminement. Nous voudrions rappeler ici quelques-unes de ses réflexions qui nous semblent primordiales : «La reconstitution romanesque de sociolectes véritables a tendance à reposer sur l'exploitation systématique d'un nombre limité de particularités verbales réelles - ou données pour telles placées moins sous l'égide de la mimésis que sous celle de la cohérence interne du texte. [...] Les transcriptions sociolectales romanesques sont en général doublées de partis pris auctoriels relativement explicites qui en assurent la légitimation et appellent, a priori, un traitement textuel aussi fidèle au réel que possible. Toutefois, force nous est d'admettre que, quel que soit le taux de "saturation sociolectale» visé, et quel que soit le niveau linguistique auquel se distribuent les marqueurs réalistes (phonétique, syntaxique, lexical), ces derniers ne sauraient se soustraire aux réductions qui caractérisent toute reproduction de la parole dans le genre romanesque.»(Lane-Mercier, 1990, pp. 44-45; gras de nous.) 
vie, par la prononciation et la syntaxe. Les structures profondes de la langue restent inchangées ${ }^{22}$.

Fondamentales à notre cheminement, les observations précédentes soulignent d'une part la responsabilité restreinte de la mimésis verbale à l'égard du réel, puisque celle-ci est nécessairement inféodée à la lisibilité; d'autre part l'importance des concepts de «clôture» et de "systématisation», que nous percevons comme les agents pragmatiques de cette lisibilité. Si la clôture marque nécessairement l'arbitraire du tri entre toutes les potentialités mimétiques, la systématisation, elle, jalonne le procédé par la reprise des mêmes indices et tempère la mimésis qui, autrement, pourrait aisément sombrer dans l'inintelligible.

Ce sera donc là le premier paramètre que nous appliquerons à notre démarche: celui d'une lisibilité symétrique en texte d'arrivée (TA). Si, comme nous l'avons déjà indiqué, le VNA n'entrave pas la lecture outre mesure, la créativité traductrice évitera à l'unisson de succomber à une représentation trop élaborée, trop scientifique $^{23}$, ou de céder à l'attrait d'un créole précis,

22. Cette dernière remarque s'applique surtout à la représentation fictionnelle du VNA.

23. Ce que nous appelons scientifique (ou scientificité) consiste à dépendre trop rigoureusement des études effectuées sur les créoles. Cette «scientificité» peut séduire abusivement le traducteur qui, établissant alors des équivalences systématiques, que cautionnent les grammaires, lexiques et dictionnaires spécialisés, risque d'aboutir à une transcription qui ne se mariera pas nécessairement avec la littérarité. Ainsi de l'énoncé «Nommes-yé rivé hier» qui, s'il est garanti par le créologue James F. Broussard (1942), pour rendre «les hommes sont arrivés hier» (yé $=$ les, ces; nomme $=$ homme; rivé= passé de arriver) peut désarçonner le lecteur. Ou celui-ci, encore plus ardu : «Moin-meinme meinme, mté camper douvant bayière ha»", lui-même entériné par une grammaire créole (Jules Faine, 1937, p. 195) pour traduire «Quant à moi, j'étais devant la barrière». 
indéchiffrable pour le lecteur ordinaire non-initié à son maniement. Ce constat élimine d'emblée un idiome complet tel que le haïtien qui, s'il a l'avantage d'être un des signes de la négritude française des Amériques, présente par contre l'inconvénient d'une grammaire et d'un lexique fixés, en outre délimités par des frontières trop «nationales $\left.{ }^{24}\right\rangle$. Un simple essai permet immédiatement de se rendre compte de l'écart qu'il imposerait par rapport à la lisibilité initiale. Soit la traduction en haitien de $\mathrm{H}-1$ :

Qui sa li vin fé la enco' avec bout touèl sa yo souli? Li paka join ou rad pou li mète souli? Koté rob satin blé que li té gain su' li lé que lité kité? Koté tout kob mari li a té gain in léli mouri é que li quité pouli a. Qui sa vié gran moun karant ans ça ap fait avec chévé li lagé sou doli tancou ou jin tifi? Qui bo li quité jin ti brail sa que lité pati avec lia. Malgré li ta prali marié? Qui koté li quité li, tove'm rache'm, que li pati avec ou soi disan ti fi tellemen jin qué li paté min guin chevé. Pouki sa li pa rété nan rol li.

Selon ce principe de «scientificite», le passage suivant de The Color Purple : «Last spring after little Lucious come I heard them fussing», pour lequel Mimi Perrin propose : «Au printemps après que le petit Lucious il est né, j'ai entendu le père et la mère se chamailler) pourrait devenir au moyen d'équivalences symétriques repérées dans les lexiques : « L'printemps-la apè li pitit Lucious li nain, mo entendu yé popa é moma dispute yémêmes» qui, s'il a l'avantage de l'exactitude, tend néanmoins à s'éloigner de notre critère fondamental de lisibilité. On remarquera entre autres l'usage de «nain» pour «être né» qui nécessiterait une note de bas de page si le traducteur décidait de l'inscrire dans son travail.

24. C'est pour cette dernière raison que nous écarterons également la possibilité d'une transcription en joual qui, outre qu'elle imposerait, au même titre que le haïtien ou l'Hexagonal des frontières trop précises, présenterait l'inconvénient d'être l'instrument d'une minorité francophone blanche, ce qui nous ramenerait donc à la problématique initiale de l'annexionnisme, que nous condamnions. 
Le lecteur, dérouté, perd vite le fil de son attention et seule s'impose à son esprit la conscience d'un code totalement autre, qui aurait nécessité de sa part un apprentissage antérieur, ce qui n'était pas le cas en TD.

Toutefois, gardons-nous bien à l'inverse de rejeter catégoriquement et entièrement le haïtien. $\mathrm{Si}$ son adoption méthodique mène à la nécessité d'un savoir préacquis, il n'en constitue pas moins dans sa systẻmaticité même une de nos ressources les plus précieuses en nous léguant un arsenal complet d'indices linguistiques, dont nous pourrons peut-être tirer parti. Car notre tâche consistera avant tout, en fonction du cadre que nous venons de délimiter, à sélectionner les marqueurs de la négritude les plus suggestifs pour parvenir à la potentialisation maximale d'un mouvement et d'une évocation analogues à ceux de nos textes de départ.

\section{Enjeux socio-historiques}

Cependant, sur le chemin de cette reconnaisance du VNA, surgit fatalement l'invocation de la non-coïncidence des cultures, celle même qui a conduit nombre de traducteurs à adopter les palliatifs que nous connaissons - après avoir invoqué la maldonne originelle de toute traduction incapable de restituer l'intégralité du réseau de connivences et de références de l'original. Or, il nous semble que cette non-coïncidence vaut la peine d'être reconsidérée à son tour puisque, dans la problématique qui est la nôtre, elle n'est pas aussi irrévocable que l'on pourrait choisir de le penser. Le français n'a-t-il pas vécu, tant en Afrique que plus tard dans les plantations américaines, une histoire qui a fait de lui une langue esclavagiste selon les mêmes modalités et les mêmes itinéraires que l'anglais, avec des résultats analogues? 
Car langue esclavagiste, le français le fut certainement ${ }^{25}$. Mais à la différence de l'anglo-américain, qui a vu les sabirs et créoles issus de son métissage prendre souche puis évoluer sur son propre territoire, le français, lui, a vécu son métissage à distance, en des lieux qui sont aussi étrangers à son identité que peuvent l'être ceux de l'anglo-américain. Un éloignement qui lui a d'ailleurs fort commodément permis de négliger cette phase de son évolution, et qui nous autorise aujourd'hui à poser le problème du décentrement non seulement en termes linguistiques mais surtout, et à proprement parler, en termes géographiques. De sorte que grâce à cette portion de leur histoire que l'anglais et le français partagent, nous pouvons orienter notre recherche vers des territoires, et suivant un itinéraire, inspirés de ceux du «commerce du bois d'ébène». Aussi, après avoir abandonné les rives de l'Hexagone, nous autoriserons-nous un élargissement du français et considérerons-nous les pays africains d'expression française comme le lieu d'une première potentialité le substrat africain du VNA et des créoles justifiera dans ce cas le lien établi avec l'Afrique.

Hélas, nous nous trouvons immédiatement confronté à un problème de taille: l'appréhension d'une équivalence. En effet, si nous considérons la production romanesque des pays d'Afrique occidentale, force nous est d'admettre que leur littérature, aussi multiple soit-elle dans ses variétés nationales, a néanmoins souvent adopté à l'égard des langages sociaux une attitude qui n'est pas sans

25. Rappelons-nous qu'il a figuré en très bonne place auprès du portugais, de l'espagnol, de l'anglais et du néerlandais sur les cartes maritimes du commerce triangulaire - dans lequel toutes les nations européennes ont d'ailleurs trempé, y compris les petites nations telles que la Suède et le Danemark. Au début, ce furent les Anglais et les Hollandais qui dominèrent, la traite française ayant démarré tard (1673) et sur une petite échelle. Mais aux XVII et $\mathrm{XVIII} \mathrm{e}^{\mathrm{e}}$ siècles, la hiérarchie des nations pratiquant la traite évolue, et au XVIII s. la France dépasse de loin la Hollande et devient, après l'Angleterre, la deuxième nation de traite des Noirs (Meyer, 1986, pp. 23-31). 
évoquer celle de la France à l'égard des siens ${ }^{26}$. Les dialogues y revêtent pour la plupart une correction académique dont est écartée toute forme vernaculaire, le purisme défensif de leurs auteurs expliquant cette éviction ${ }^{27}$. Apparaîtra parfois un personnage au parler idiomatiquement coloré, mais ces interventions sont rares, et servent à préciser une distinction sociale précise, la plupart du temps d'infériorité. La rareté du phénomène n'enlève cependant rien à son utilité puisque, au même titre que le haîtien, il attire notre attention sur les marqueurs idiolectaux utilisés. Ainsi de l'extrait suivant :

Bokossa, un tirailleur dahoméen, incapable de contenir sa douleur, éleva sa voix d'illettré dans une pathétique oraison funèbre:

«Aurivoi camara, aurivoi Houngbé! Tu dis qué tu n'as plus pêsonne au Danhomin, mais Danhomin est glan et le monde aussi est très glan; alo' peut-et', j'irai voi ton pê ou ta mê ou ta sœu' ou quêqu'un des tiens au pays. C'est alo' qué jé lui dilai qué tu n'es pas mort à la guerre, ni à Dien-Bien-Phu, ni à la Flance, mais ici dans lé bateau, sous lé soleil aflicain, tout d'un coup seuliment! Adié Houngbé!» termina-t-il dans un douloureux sanglot. (BhêlyQuénum, 1965, p. 31)

26. En effet, phénomène économico-politique, le colonialisme tendait à reproduire là où il se manifestait la division de classes entretenue sur les territoires métropolitains. De sorte que littérature émergeante, la production africaine s'est conformée massivement aux idéaux de la langue mère. Voir sur ce sujet l'étude de LouisJean Calvet (1974). Nous constaterons que l'un des critères les plus souvent invoqués pour évaluer la littérature franco-africaine, est en fait celui de sa correction langagière.

27. Cette correction ne doit pas nous surprendre puisque l'élite francophone africaine ne fixe pas la norme dominante, établie dans la métropole. Elle ne peut que l'imiter: d'où un sentiment d'insécurité affectant la masse des «lettrés»; d'où leur hypercorrection, puisque la légitimité de leur statut est dans une large mesure garantie par l'exactitude de cette imitation (Manassy et Wald, 1984, pp. 22-23). 
Même si, comme les propos de Bokossa semblent nous le suggérer, la possibilité existait de rassembler un appareil suffisant de procédés pragmatico-linguistiques, surgirait néanmoins un second problème: celui de la nature des marqueurs. Le doute demeurerait dans notre esprit quant à la validité des indices réunis, cela en raison $\mathrm{du}$ caractère autrement très classique des cuvres interrogées ${ }^{28}$. Jusqu'à quel point leur appréhension n'est-elle pas médiatisée par des critères inconscients, qui se conformeraient à un type de représentation accréditée depuis longtemps par la métropole (telle la fameuse élision du «r» [aurivoi'; pêsonne; alo'; ton pê ou ta mê ou ta sœu'] ou son remplacement par le «l» central [aflicain]), et qui serait plus proche de la stéréotypification que de la typification? Les auteurs ont tous subi la rigueur du «corset classique», selon l'expression de Claude Hagège (1987), et n'éprouvent pas forcément la nécessité de s'en dégager ${ }^{29}$. Quelle sera la position de la

28. Nous avons parcouru un certain nombre de romans africains qui, hélas, n'ont guère apporté d'eau à notre moulin. Comme nous le mentionnons, la norme y est encore trop prévalente, évinçant de la sorte toute forme sociolectale. Voici quelques-uns des titres sollicités: Le Vieux nègre et la médaille (1956) et Chemin d'Europe (1960) de Ferdinand Oyono; Cet «autre» de l'homme (1972) et Contes et légendes du Niger (1972) de Boubou Hama; le Chant du lac (1965) de Olympe Bhêly-Quénum; Roman sénégalais, suivi de Contes et légendes d'Afrique noire (1948) de Ousmane Soké; les Exilés de la forêt vierge ou le Grand complot (1974) de Jean-Pierre Makouta-Mboukou; le Cercle des Tropiques (1972) de Alioum Fantoure; Kocoumbo, l'étudiant noir (1960) de Aké Loba; etc.

29. D'autant que dans un pays où le français est importé, «la langue des livres apparaît comme une valeur fiable, une garantie contre toute erreur. La vénération accordée par la communauté des locuteurs natifs à ses écrivains et attestée en milieu scolaire (lieu d'apprentissage usuel) par l'admiration sans réserve des maittres, impose à des utilisateurs non-natifs et, de ce fait, peu assurés d'eux-mêmes dans le nouveau parler auquel ils s'initient, une norme du bel usage plus rigoureuse qu'elle ne l'est dans la métropole [...].» Lafage cité par Manassy et Wald (1984, p. 23). 
nouvelle littérature noire, surtout post-indépendantiste, à cet égard? Verra-t-on l'émergence d'un mouvement d'émancipation analogue à celui du VNA aux États-Unis ou du joual au Québec? Une étude récente sur le roman africain (Pius Ngandu Nkashama, 1989) n'envisage pas la problématique du rapport entre langue normative et langue créolisante dans la mimésis verbale, et ne peut nous fournir d'éléments de solution puisque, traitant avant tout de l'inscription idéologique dans le discours narratif, elle n'envisage la parole que dans son contenu, jamais selon sa forme, ni dans les confrontations qui pourraient en résulter. Tout n'est peut-être pas dit à propos de l'Afrique francophone mais, pour l'instant, ce n'est pas de sa fiction romanesque (du moins dans la connaissance que nous en avons) que pourra venir la solution.

À l'autre pôle de notre itinéraire, la plantocratie américaine, qui, comme nous le savons, a vu naître le Gombo de la Louisiane ou créole français des anciens esclaves. Ici, le problème est inverse. Nous n'avons plus affaire au souci de pureté d'une littérature en voie d'affirmation, mais à la pénurie de ressources d'une littérature moribonde. Avec la fin du XIX ${ }^{\mathrm{e}}$ siècle, le français, à toutes fins pratiques, a cessé d'exister «in Louisiana». Seuls nous restent en 1990 les souvenirs d'une culture révolue ${ }^{30}$ et quelques îlots de survivance, où le gombo partage avec le cajun son espace vital. Situation d'autant plus navrante que nous nous situions sous les mêmes cieux que l'anglais; que la langue y avait subi des

30. Nous ne pouvons manquer de mentionner en passant la remarquable chronique romancée de Maurice Denuzière sur la Louisiane: Louisiane, Fausse-Rivière, Bagatelle, les Trois-Chênes, l'Adieu au Sud et les Années Louisiane. Tentive contemporaine de restituer fictivement un peu de ce passé perdu. Le travail de $M$. Denuzière nous a paru digne d'intérêt pour la richesse de sa documentation sur le dernier siècle du français en territoire américain. La situation des Noirs dans l'œuvre y est, on s'en doute, symptomatique de leur position sociale : ils n'y occupent qu'un espace diégétique restreint, et leurs rares paroles égrènent les procédés mimétiques habituels (principalement l'élision du «r»). 
déformations parfaitement comparables; qu'elle y avait vécu des enjeux analogues, et que la coïncidence des cultures invoquée au début de ce chapitre y était quasiment réalisée.

Certains textes demeurent quand même, fixés au XIX ${ }^{e}$ siècle ou dans la première moitié du $\mathrm{XX}^{\mathrm{e}}$, auxquels il est possible de faire appel. Ainsi de l'ouvrage de James F. Broussard, Louisiana Creole Dialect, ou de celui de Gérard Labarre St. Martin et Jacqueline K. Voorhies, Écrits louisianais du dix-neuvième siècle, dont nous avons pu nous inspirer pour la formation d'un mini-lexique ${ }^{31}$. Il était dès lors possible d'élaborer la transcription suivante de $\mathrm{W}-1$ :

\section{Cher Bon Djé}

Mo moman lé morte.

Li mort pas contente contre moin.

Li guélé apé moin.

Mo grosse.

Mo li pas capab aller vite.

Ca fait quand mo revini du puits-la, l'eau li té chaude.

Quand mo préparé le manger, le manger li té froid.

Quand mo préparé yé zenfants pou' l'école, l'est déjà

l'heure apé dîner.

Mo popa li dit rien.

Li assi-là côté so lit. Li tient so main-la. Li pleuré. Li dit : «To pas quitté moin, to ni pas allé.»

Toutefois, l'entreprise nous a paru bien artificielle : nous nous évertuions à traduire dans un idiome défunt toute l'énergie d'un autre en pleine expansion et, dans le cas de Zora Neale Hurston, nous risquions de voir s'estomper l'intention première de notre projet : restituer un peu de la vivacité de son vernaculaire.

Aussi est-ce pour cette dernière raison que nous écrivions que tout n'était pas dit à propos de l'Afrique francophone. Elle

31. On se reportera à l'appendice pour le lexique et sa mise en application. 
constitue le nouveau bassin de créolisation, le lieu d'un nouveau ferment qui paraît contenir plus de promesses.

Ce processus, que nous avons ailleurs désigné quelque peu arbitrairement par le terme de "vernacularisation», a pour effet manifeste de restituer au code utilitaire à quoi se réduit dans la pratique le français des non-lettrés la flexibilité stylistique qui lui fait défaut. [...] Du point de vue linguistique on peut y voir l'amorce d'une évolution qui conduirait à la constitution d'un créole si ce qui n'est encore que conventions de langage se trouvait incorporé à la grammaire du parler. (Manassy et Wald, 1984, p. 45)

Les journaux, les bandes dessinées, les films, les pièces et la nouvelle fiction de la francophonie africaine devraient à l'avenir pouvoir subvenir aux besoins du vernaculaire noir américain. $\mathrm{Ne}$ serait-ce pas là l'application de la traduction non-ethnocentrique que préconise Berman?

\section{Bibliographie}

ANGENOT, Marc (1979). Glossaire pratique de la critique contemporaine. Québec, Éditions Hurtubise/HMH.

ASTINGTON, Eric (1983). Equivalences: Translation. Difficulties and Devices. Cambridge, Cambridge University Press.

BAKER, Houston A. (1984). Blues, Ideology, and Afro-American Literature: A Vernacular Theory. Chicago, University of Chicago Press.

BAR-TAL, Daniel, Carl F. GRAUMANN, Arie W. KRUGLANSKI et Wolfgang STROEBE, dir. (1989). Stereotyping and Prejudice: Changing Conceptions. New York, Springer-Verlag New York Inc. 
BARTHES, Roland (1953). Le Degré zéro de l'écriture. Paris, Gallimard.

IV. Paris, Seuil.

(1984). Le Bruissement de la langue. Essais critiques

BERMAN, Antoine (1984). L'Épreuve de l'étranger: Culture et traduction dans l'Allemagne romantique. Paris, Gallimard.

(1985). «La Traduction comme épreuve de l'étranger», Texte: Traduction/Textualité, 4 , pp. 67-81.

(1989). «La Traduction et ses discours», Meta, XXXIV(4), pp. 672-679.

BHÊLY-QUÉNUM, Olympe (1965). Le Chant du lac. Paris, Éditions Présence africaine.

BOGLE, Donald (1989). Toms, Coons, Mulattoes, Mammies, and Bucks: An Interpretive History of Blacks in American Films. (Édition revue et élargie). New York, The Continuum Publishing Company.

BOURDIEU, Pierre (1982). Ce que parler veut dire. L'économie des échanges linguistiques. Paris, Fayard.

BRAXTON, Joanne M. (1989). Black Women Writing Autobiography: A Tradition within a Tradition. Philadelphia, Temple University Press.

BROUSSARD, James F. (1942). Louisiana Creole Dialect. c1942 by Louisiana State University Press. $2^{\mathrm{e}}$ éd.: Port Washington, New York et Londres, Kennikat Press, 1972.

CABAU, Jacques (1966). La Prairie perdue. Le roman américain. Paris, Seuil. Nouvelle édition, Points, 1981. 
CABLE, George W. (1902). Old Creole Days. Edinburgh, David Douglas.

CALVET, Louis-Jean (1974). Linguistique et colonialisme. Petit traité de glottophagie. Paris, Petite bibliothèque Payot.

CHRISTIAN, Barbara (1989). «But What Do We Think We're Doing Anyway: The State of Black Feminist Criticism(s) or My Version of a Little Bit of History", in C. A. Wall, dir. (1989), pp. 58-74.

COUSINS, Paul M. (1968). Joel Chandler Harris, A Biography. Baton Rouge, Louisiana State University Press.

DILlARD, J., dir. (1975). Perspectives on Black English. The Hague, The Netherlands, Mouton \& Co.

DILLARD, J. L. (1973). Black English. Its History and Usage in the United States. New York, Vintage Books Edition.

DUCHET, Claude, dir. (1977). Sociocritique. Paris, Nathan.

DUCROT, Oswald (1980). Les Mots du discours. Paris, Les Éditions de Minuit.

FAINE, Jules (1937). Philologie créole. Études historiques et étymologiques sur la langue créole d'Haiti. Port-au-Prince, Imprimerie de l'État.

FAULKNER, William (1954).The Sound and The Fury. New York, Random House, Vintage Books. [1 $1^{\text {ire }}$ éd.: New York, Jonathan Cape and Harrison Smith, 1929]

FOX-GENOVESE, Élisabeth (1988). Within the Plantation. Black and White Women of the Old South. Chapel Hill \& London. The University of North Carolina Press. 
GERMAIN, Robert (1988). Grammaire créole. Paris, Éditions L'Harmattan.

GEORGE, Nelson (1989). The Death of Rhythm and Blues. New York, E. P. Dutton. Published simultaneously in Canada by Fitzhenry and Whiteside, Limited, Toronto.

GLOSTER, Hugh M. (1948). Negro Voices in American Fiction. New York, Russell \& Russell, 1965. First published by the University of North Carolina Press.

GOUNARD, Jean-François (1984). Le Problème noir dans les cuvres de Richard Wright et de James Baldwin. Sherbrooke, Éditions Naaman.

GRAY, Richard (1977). The Literature of Memory, Modern Writers of the American South. Baltimore \& London, The Johns Hopkins University Press.

GRESSET, Michel (1985). «Aspects de la traduction littéraire: Poe et Baudelaire, Faulkner et ses interprètes», SSLM-Annuario, 1, Trieste.

GROUPE $\mu$ (1982). Rhétorique générale. Paris, Seuil, Points.

GUIRAUD, Pierre (1980). L'Argot. Paris, PUF, Que sais-je?

HAGÈGE, Claude (1985). L'Homme de paroles. Contribution linguistique aux sciences humaines. Paris, Folio, Essais, Arthème Fayard.

Jacob.

(1987). Le Français et les siècles. Paris, Éditions Odile

HENDERSON, Mae Gwendolyn (1989). «Speaking in Tongues: Dialogics, Dialectics, and the Black Woman Writer's Literary Tradition", in C. A. Wall, dir. (1989), pp. 16-37. 
HOLLOWAY, Karla F. (1987). The Character of the Word. The Texts of Zora Neale Hurston. Wesport, Greenwood Press Inc.

HOMEL, David et Sherry SIMON, dir. (1988). Mapping Literature. The Art and Politics of Translation. Montréal, Véhicule Press.

HOWARD, Lillie P. (1980). Zora Neale Hurston. Boston, Twayne Publishers.

HURSTON, Zora Neale (1937). Their Eyes Were Watching God. [1 $1^{\text {ere }}$ éd.: J. B. Lippincott, Inc.] New York, Harper \& Row Publishers, Perennial Library, 1990.

(1935). Mules and Men. [1 ${ }^{\text {ère }}$ éd.: J. B. Lippincott, Inc.] New York, Harper \& Row Publishers, Perennial Library, 1990.

(1981). The Sanctified Church. The Folklore Writings of Zora Neale Hurston. Toni Cade Bambara, ed. Berkeley, Turtle Island Foundation.

JOACHIM, Sébastien (1980). Le Nègre dans le roman blanc: lecture sémiotique et idéologique de romans français et canadiens, 19451977. Montréal, Presses de l'Université de Montréal.

LABARRE, Gérard et Jacqueline K. VOORHIES, dir. (1979). Écrits louisianais du dix-neuvième siècle. Nouvelles, contes et fables. Baton Rouge and London. Louisiana State University Press.

LABOV, William (1976). Sociolinguistique. Paris, Éditions de Minuit.

LANE-MERCIER, Gillian (1990). «Pour une analyse du dialogue romanesque», Poétique 81, pp. 43-62.

LEHISTE, Ilse (1988). Lectures on Language Contact. Cambridge and London, The MIT Press. 
LOEWENBERG, Bert James and Ruth BOGIN (1976). Black Women in Nineteenth-Century American Life. Their Words, Their Thoughts, Their Feelings, The Pennsylvania State University Press. University Park and London.

MAGNY, Claude-Edmonde (1950). Histoire du roman français depuis 1918. Paris, Seuil, Points.

MAGUIRE, Robert E. (1979). Notes on Language Use Among English and French Creole Speaking Blacks in Parks, Louisiana. Projet Louisiane, Document de travail $n^{\circ} 6$, juillet 1979. InterAmerican Foundation, Rosslyn Virginia.

MANASSY, Gabriel et Paul WALD (1984). Le Français en Afrique noire, tel qu'on le parle tel qu'on le dit. Paris, L'Harmattan IDERIC.

McCRUM, Robert, William CRAN and Robert MACNEIL (1986). The Story of English. New York, Elisabeth Sifton Books - Viking, Viking Penguin Inc.

McMILLAN REEVES, George, dir. (1971). The Time of William Faulkner. Essays by Maurice-Edgar Coindreau, with a Foreword by Michel Gresset. Columbia, University of South Carolina Press.

MEISEL, Jurgen M., dir. (1977). Langues en contact - Pidgins Créoles -Languages in Contact. Tübingen, TBL-Verlag Narr.

MESCHONNIC, Henri (1973). Pour la poétique II. Épistémologie de l'écriture. Poétique de la traduction. Paris, Gallimard.

MEYER, Jean (1986). Esclaves et Négriers. Paris, Gallimard, Découvertes.

NETTELS, Elsa (1988). Language, Race, and Social Class in Howells's America. Lexington, The University Press of Kentucky. 
NYSSEN, Hubert, dir. (1986). Actes des deuxièmes assises de la traduction littéraire (Arles 1985). Arles, Atlas, Actes Sud.

dir. (1987). Actes des troisièmes assises de la traduction littéraire (Arles 1986). Arles, Atlas, Actes Sud.

PELLETIER, Jacques, dir. (1984, 1985). Le Social et le littéraire. Montréal, Université du Québec à Montréal, Cahiers du Département d'études littéraires, $2^{\mathrm{e}}$ éd.

PERRIN, Mimi (1986). «Improviser comme les jazzmen», Actes des deuxièmes assises de la traduction littéraire (Arles, 1985). Arles, Atlas, Actes sud.

PETRY, Alice Hall (1988). A Genius in His Way. The Art of Cable's Old Creole Days. Associated University Press Inc.

PIUS NGANDU NKASHAMA (1989). Écritures et discours littéraires. Études sur le roman africain. Paris, Éditions L'Harmattan.

RAIMOND, Michel (1976). Le Roman contemporain: le signe des temps. Paris, Société d'édition d'enseignement supérieur.

SAPORTA, Marc (1976). Histoire du roman américain. Paris, Gallimard, nouvelle édition.

SARTRE, Jean-Paul (1948). Qu'est-ce que la littérature? Paris, Gallimard, Folio essais.

SHOCKLEY, Ann Allen (1988). Afro-American Women Writers 1746-1933. An Anthology and Critical Guide. Boston, G. K. Hall \& Co.

TERRIER, Michel (1973). Individu et société dans le roman américain de 1900 à 1940. Essai de poétique sociale. Paris, Librairie Marcel Didier. 
TISCHLER, Nancy M. (1969). Black Masks. Negro Characters in Modern Southern Fiction. University Park and London, The Pennsylvania State University Press.

VALDMAN, Albert, dir. (1979). Le Français hors de France. Paris, Éditions Honoré Champion.

VIATTE, Auguste (1954). Histoire littéraire de l'Amérique française, des origines à 1950. Paris, Presses Universitaires de France. Québec, Presses de l'Université Laval.

WALL, Cheryl A., dir. (1989). Changing Our Own Words. Essays on Criticism, Theory, and Writing by Black Women. New Brunswick and London, Rutgers University Press.

WALKER, Alice (1982). The Color Purple. New York, Harcourt Brace Jovanovich.

(1984). In Search of Our Mothers' Gardens. Harvest/HBJ. [ ${ }^{\text {ère }}$ éd.: New York, Harcourt Brace Jovanovich, 1983].

WALTER, Henriette (1988). Le Français dans tous les sens. Paris, Robert Laffont.

WARDHAUGH, Ronald (1986). An Introduction to Sociolinguistics. Oxford and New York, Basil Blackwell Ltd.

WELLEK, René et Austin WARREN (1971). La Théorie littéraire. Paris, Seuil.

ZÉRAFFA, Michel (1971). Roman et société. Paris, Presses Universitaires de France.

ZIMA, Pierre V. (1985). Manuel de sociocritique. Paris, Picard éditeur. 


\section{Appendice}

Traduction du segment initial H-1.

Les navires au loin emportent à leur bord les espoirs de chaque homme. Pour certains, ils entrent au port avec la marée. Pour les autres, ils croisent éternellement à l'horizon, toujours à portée de vue, n'accostant jamais, jusqu'à ce que le Guetteur, résigné, détourne son regard, ses rêves mortifiés par le Temps. Telle est la vie des hommes.

Pour leur part, les femmes oublient tout ce dont elles ne veulent pas se souvenir, et se souviennent de tout ce qu'elles ne veulent pas oublier. Le rêve est la vérité. Ensuite elles passent aux actes et agissent conformément.

Une femme se trouve au début de ce récit. Elle revenait d'enterrer les morts. Non pas ceux qui meurent d'une longue maladie, entourés de leurs amis à leur chevet. Non. Elle revenait de ces morts abrutis et gonflés; ceux qui meurent sans qu'on s'y attende, les yeux grand ouverts, accusateurs.

Tout le monde la vit revenir : c'était à l'instant du couchant. Le soleil avait disparu, laissant tarder sa trace dans le ciel. C'était l'heure où l'on s'assoit sur la galerie, en bordure de la route. C'était l'heure où l'on écoute et discute. Eux qui étaient maintenant assis, n'avaient été la journée durant que des commodités, dépourvues de langue, d'oreilles et d'yeux. La brute et le mulet avaient occupé leur peau. Mais maintenant que le soleil et le patron avaient disparu, leur chair retrouvait sa force et son humanité. Ils se transformaient en seigneurs de paroles et autres moindres choses. Ils échangeaient des nations par la bouche. Juges, ils siégaient.

De la revoir telle qu'elle était, leur rappela toute l'envie qu'ils avaient accumulée en d'autres occasions. Ils remâchèrent leurs idées de derrière la tête et déglutirent de plaisir. De leurs questions ils firent des affirmations brûlantes, de leurs rires des instruments meurtriers. C'était une cruauté collective. Un sentiment revenu à la vie. Des paroles libres, marchant sans maîtres, marchant à l'unisson comme dans l'harmonie d'une chanson.

"Qu'est-ce que li révini fé la enco' dans so costume-la? Li pas capab trouvé une robe pou' s'mettre sur li? - Qu'est-ce qu'elle a fé avé sa rob-la de satin bleu que li apré porter quand li quitté. - Qu'est-ce qu'elle a fé avé tout so l'argent-la so nhomme li gagné et li donné quand li mouri? - Qui ça ein grande femme-la de quarante ans li apé fé avé so cheveux-la qui ça 
li pend dans so dos comme si li té enco' ein jeune femme-la. — Qui ça fé de li jeune boug'e-la que li parti avec? - Si to crois que li allé se marier avé lui. — Où c'est qu'il li laissé tomber? - Qui ça qu'il a fé avé tout so l'argent-la? - To dit qu'il est parti avé une aut' jine fille. - Pourquoi ça que li reste pas avec ceux de so classe-la?»

Parvenue à leur hauteur, elle tourna la tête, à la barrière enrondins, et leur parla. Ils bredouillèrent un "bonsoi'» sonore, la bouche ouverte, les oreilles avides. Ses paroles étaient certainement plaisantes, mais elle ne s'arrêta pas et continua jusqu'à son portail. La galerie, les yeux rivés, en oubliait de parler.

RÉSUMÉ: Le vernaculaire noir américain: Ses enjeux pour la traduction envisagés à travers deux œuvres d'écrivaines noires, Zora Neale Hurston et Alice Walker - Zora Neale Hurston et Alice Walker font usage dans leurs œuvres d'une langue autre, d'un sociolecte longtemps dénigré, le vernaculaire noir américain. Cette utilisation va bien au-delà de la simple caractérisation sociale de leurs personnages et constitue un geste contestataire, une revendication et une célébration. Dans ces conditions, la traduction annexionniste qui consisterait à avoir recours à des sociolectes effaçant totalement la négritude et la problématique raciale, tel le langage «paysan», apparaît comme une véritable mutilation des œuvres. Il convient donc d'opérer le décentrement du texte-cible en y inscrivant la négritude. Les divers créoles à base française et les variétés du français parlées en Afrique noire peuvent fournir des marqueurs qui, sans relocaliser abusivement le texte-cible, serviront à cette fin.

\footnotetext{
ABSTRACT: Black English Vernacular at Issue: Translating the Work of Zora Neale Hurston and Alice Walker - Zora Neale Hurston's and Alice Walker's works are notable for their use of an Other language - the long-denigrated sociolect that is Black English Vernacular. This use goes well beyond the mere social delineation of the characters, and is at once an act of contestation, a reclamation and a celebration. Under these conditions, annexationist French translations, which resort to sociolects - "paysan" speech, for example - that completely erase both the negritude of the original and the problem of race, in fact constitute nothing less than a mutilation of the source texts. A possible solution would be to effect a decentering of the target texts by inscribing the originals' negritude therein. Markers that would serve this purpose without unduly relocating the target text are to be found in the various French language-based Creoles and in the different types of French spoken in Black Africa.
} 


\section{Mini-lexique de créole louisianais établi à partir des contes, fables et autres histoires du folklore.}

Standard

Mon homme/sa femme

Il y avait

Quand il est né

Tout le monde

Parce que

Il est revenu

Il est malade

Ils se sont mis à pleurer

Peut-être

Ils étaient tous contents

Il est tombé par terre

Aujourd'hui/demain

Il m'a quittée

Diable

Il a besoin de manger

J'ai besoin de dormir

Nous n'avons besoin de rien

Est-ce que tu peux faire ça?

Je suis capable/je peux

J'ai travaillé hier

Il s'est noyé

Tu vois bien maintenant

Toujours le plus malin

Quand ils se sont réveillés

Je suis toujours content de

manger

Parce qu'il était si petit

Je vous paierai lui dit-elle

Avant l'automne

Je vais raconter une histoire

Dis-moi qui tu aimes

Je te dirai qui tu es
Gombo

Page

Mo nomme/so femme

44

Yé té $\quad 76$

Quand li nain $\quad 76$

Tout moune $\quad 76$

ça fait $\quad 67$

Li révini $\quad 76$

Li malade $\quad 76$

Yé commencé plérer $\quad 76$

Pététe $\quad 78$

Yé té tout contents $\quad 78$

Li tommebé par té $\quad 35$

Jordi/démain 35

Li quittée moin $\quad 44$

Djabe 36

$\mathrm{Li}$ bésoin manger 29

Mo bésoin dormir 29

Nous bésoin de rien 29

To capab fait ça? 19

Mo capab

Mo travaillé hier

Li néyé li-même $\quad 74$

Ta oua bein asté $\quad 74$

Toujou' plis smatte $\quad 74$

Quand yé réveillé 72

Mo toujou' content manger

À cause li té si piti $\quad 65$

M'a payer toi, li dit $\quad 88$

Avant l'ôtonne $\quad 88$

M'a reconter ein nistwa' $\quad 110$

Dis-moi qui to lainmain 35

M'a dit toi qui to y 35 